\title{
Editorial
}

\section{A new model for clinical trials to address the COVID-19 emergency}

The COVID-19 pandemic represents the most serious health and economic catastrophe in our lifetimes. The heartfelt and urgent call to arms from Epelbaum and Galperin [1] in this issue of Breathe reflects the harsh reality for those on the frontline: the medical imperative to make decisions in the context of imperfect information. One of the unique features of this crisis is that we are dealing with a novel coronavirus. We are starting from a blank sheet in terms of empirical data on which to base decisions about interventions for prevention and treatment. Clinicians need to make the best use of the imperfect information at the bedside. However, this is not the end of the story; indeed, it is the beginning. In history, now and in the future, research has been, and is, the method we use to acquire knowledge and knowledge is the key to effective action to solve clinical problems. Research is the key to solving the COVID-19 emergency.

There are some special features of the COVID19 pandemic. Most problems are solved using a fund of existing knowledge, accrued over decades, augmented from time to time in small or large increments. The existing research model is designed for, and well adapted to, this purpose. The COVID-19 pandemic represents a different type of problem. First, as noted above, it is a novel coronavirus. Initial decisions were based on analogy with similar viruses (SARS, MERS, influenza, etc.) and other infections (tuberculosis). Over time, this was augmented by case reports [2, 3], case series [4], and small-scale [5, 6] and then a few larger clinical trials $[7,8]$. However, there is no large corpus of knowledge to build upon. Second, it is a global pandemic with catastrophic health and socioeconomic consequences. The pressure to find solutions quickly is intense but resources, financial, human, research participants and other, are limited, and prioritisation in the allocation of these resources is important to ensure that they are used efficiently and effectively. It is crucial that interventions are implemented based on robust evidence and not rushed into clinical practice without strong evidence [9].

These special features of the COVID-19 pandemic mean that the several aspects of the "business-as-usual" approach to research are not fit for purpose. First, competition among researchers and research organisations is productive in the normal course of research. However, in the present context, this competition only serves to inhibit collaboration and transparency, both of which are critical to the rapid, efficient and effective generation of knowledge. Second, the existence numerous small trials competing for the same pool of participants and trials that recruit only a minority of patients with the target condition, due to restrictive eligibility criteria and a limited range of clinical entry points or trial sites, are both inefficient and ineffective. These trials often do not achieve their endpoints in a timely manner and fail to provide sufficiently generalisable information to guide implementation. This problem is exacerbated when trials are not harmonised: having different selection criteria, endpoints and reporting methods. Third, the practice of commencing translation into
Cite as: Marks GB. A new model for clinical trials to address the COVID-19 emergency. Breathe 2020; 16: 200220. 
practice only after completion of recruitment and publication means that many patients do not get access to optimal modes of treatment in a timely manner. This is especially pertinent given the rapid evolution of the COVID-19 situation and the urgent need for effective interventions. Hence, there are serious problems in attempting to meet the urgent need for solutions to COVID-19 using the existing model for organising clinical trials.

We need a new model for coordination, prioritisation and implementation of research that is fit-for-purpose for the COVID-19 pandemic. An effective model would include three key components. First, a constantly updating and testable causal model for COVID-19 that is designed to identify and prioritise potential interventions to prevent, mitigate and cure the disease, and to target specific interventions to the most appropriate risk groups. Second, a pipeline of pharmacological and nonpharmacological interventions for prevention, mitigation and cure, linked to the causal model, that are suitable for testing in randomised controlled trials. Third, a clinical trials platform and information system linked to people at risk of, and diagnosed with, COVID-19, which ensures that all such people receive the optimal treatment for their condition, where this is established, or are randomly allocated alternative treatments most likely to be safe and effective based on their clinical and epidemiological characteristics and the current state of the evidence.

Establishing consensus around the causal model for COVID-19 is challenging in the environment of rapidly evolving, but limited, information. An evidence- and expert-elicitation process is required. This process includes systematic synthesis of relevant evidence; establishing a balanced and independent panel of experts from the relevant range of clinical, scientific, management and consumer perspectives; eliciting consensus around a disease logic (that is, causal) model for COVID19; and eliciting expert decisions based on the synthesis of evidence and the agreed logic model. These last two steps utilise expert-elicitation tools including Bayesian networks, logic maps and a modified Delphi process [10, 11]. This is an ongoing, constantly updating process, so that the causal model always reflects consensus around the most recent available evidence. The causal model provides a framework for prioritising interventions to test in clinical trials.

A clinical trials platform based on a Bayesian adaptive "decision-engine" is needed for making treatment recommendations for individual patients to allow research to be seamlessly integrated with research translation [12, 13]. This is designed to simultaneously:

- contribute to the generation of knowledge by testing the best candidate interventions;

- adapt trial designs to the rapidly evolving knowledge base (both internally and externally generated); and

- ensure, in this rapidly evolving knowledge field, that each patient receives the best possible treatment at the time they require it.

A central feature of the Bayesian design is the capacity to compare effectiveness in an ongoing manner, during the conduct of the trial. New treatments can be rapidly implemented based on accelerated acquisition of evidence about the effectiveness and safety. This Bayesian adaptive decision engine ensures that treating clinicians receive, for each patient, either:

- a recommendation for one or more specified treatments or for no treatment, where there is a high degree of certainty that this decision is optimal; or

- random allocation to one of two or more treatments, where there is uncertainty about the optimal treatment.

This model of trial design is ideal for managing the COVID-19 emergency.

We must rise to the challenge of the COVID19 pandemic. Our personal and clinical lives have changed profoundly. Research is the key to solving this problem and the world of research must adapt to solve this crisis. Old ways are not fit-for-purpose. We need to re-imagine the way we do research.

\section{Affiliations}

\section{Guy B. Marks 1,2}

${ }^{1}$ South Western Sydney Clinical School, University of New South Wales, Sydney, NSW, Australia. ${ }^{2}$ Woolcock Institute of Medical Research, University of Sydney, Camperdown, NSW, Australia.

\footnotetext{
Acknowledgements

I acknowledge helpful comments by Kerrie Mengersen (Queensland University of Technology, Brisbane, Australia), Ben Marais, Thomas Snelling (both University of Sydney, Sydney, Australia) and Jennifer Martin (University of Newcastle, Newcastle, Australia) on an earlier version of this manuscript.
} 


\section{Conflict of interest}

G.B. Marks reports a grant paid to his institution by and participation in an advisory board for AstraZeneca, and a grant paid to his institution by GSK, outside the submitted work.

\section{References}

1. Epelbaum O, Galpein I. In defence of extrapolation but not improvisation in SARS-CoV-2 lung disease. Breathe 2020; 16 : 200113.

2. Holshue ML, DeBolt C, Lindquist S, et al. First case of 2019 novel coronavirus in the United States. N Engl J Med 2020; 382: 929-936.

3. Xu Z, Shi L, Wang Y, et al. Pathological findings of COVID-19 associated with acute respiratory distress syndrome. Lancet Respir Med 2020; 8: 420-422.

4. Guan WJ, Ni ZY, Hu Y, et al. Clinical characteristics of coronavirus disease 2019 in China. N EnglJ Med 2020; 382: 1708-1720.

5. Cao B, Wang Y, Wen D, et al. A trial of lopinavir-ritonavir in adults hospitalized with severe Covid-19. N EnglJ Med 2020; 382: 1787-1799.

6. Aronson JK, Ferner RE, DeVito N, et al. COVID-19 trials registered up to 8 March 2020 - an analysis of 382 studies. Oxford, Centre for Evidence-Based Medicine, 2020.www.cebm.net/ oxford-covid-19/covid-19-registered-trials-and-analysis/.

7. Wang Y, Zhang D, Du G, et al. Remdesivir in adults with severe COVID-19: a randomised, double-blind, placebo-controlled, multicentre trial. Lancet 2020; 395: 1569-1578.
8. Group RC, Horby P, Lim WS, et al. Dexamethasone in hospitalized patients with Covid-19 - preliminary report. $N$ Engl J Med 2020; in press [http://doi.org/10.1056/ NEJMoa2021436].

9. Angus DC. Optimizing the trade-off between learning and doing in a pandemic. JAMA 2020; 323: 1895-1896.

10. Stewart GB, Mengersen K, Meader N. Potential uses of Bayesian networks as tools for synthesis of systematic reviews of complex interventions. Res Synth Methods; 5: 1-12.

11. Martin TG, Burgman MA, Fidler F, et al. Eliciting expert knowledge in conservation science. Conserv Biol; 26: 29-38.

12. Gajewski BJ, Berry SM, Quintana M, et al. Building efficient comparative effectiveness trials through adaptive designs, utility functions, and accrual rate optimization: finding the sweet spot. Stat Med 2015; 34: 1134-1149.

13. Wason JM, Trippa L. A comparison of Bayesian adaptive randomization and multi-stage designs for multi-arm clinical trials. Stat Med 2014; 33: 2206-2221. 